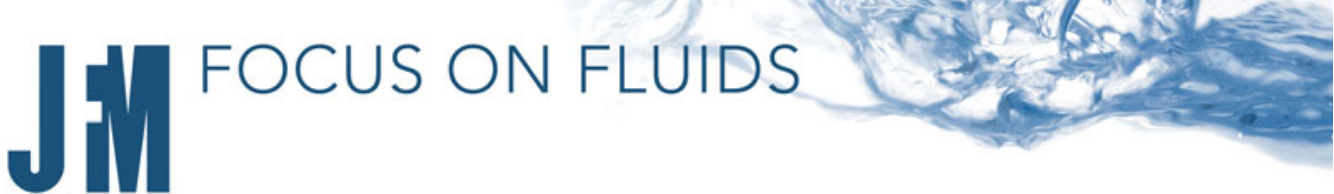

\section{The impulsive swirl of a gas}

\author{
John Elie Sader $\dagger$ \\ School of Mathematics and Statistics, The University of Melbourne, Victoria 3010, Australia
}

(Received 27 November 2020; accepted 27 November 2020)

The motion of a sphere in a viscous gas has been studied since the time of Sir George Gabriel Stokes who explored linear, steady and unsteady flows. While the unsteady Stokes equation is often used to calculate these flows, this continuum treatment cannot capture some key physical phenomena. This includes propulsion of a sphere by temperature gradients on its surface, without convection. Taguchi et al. (J. Fluid Mech., 2021) now calculate the flow generated by the impulsive rotation of a sphere in a gas, a problem first proposed by Stokes, using the linearised Boltzmann-BGK (Bhatnagar, Gross, Krook) equation. This statistical mechanical approach naturally captures continuum through to collisionless flows; the latter occurs even when the gas mean free path is small. The heat flow generated by the sphere is also determined - a non-continuum effect - showing its direction reverses as the flow evolves. The predicted phenomena are yet to be observed in experiment.

Key words: non-continuum effects, micro-/nano-fluid dynamics, kinetic theory

\section{Introduction}

Sir George Gabriel Stokes posed the now widely studied problem (Stokes 1851)

'Suppose now the solid to be a sphere, having its centre at the origin. Let $a$ be its radius, $\succ$ its angular velocity, and suppose the fluid initially at rest. Then [the fluid velocity] $v^{\prime}$ is to be determined from the general equation (182)...'

where (182) is the 'unsteady Stokes equation' (Kim \& Karrila 2005). At the time, Stokes calculated the long-time steady state motion of the fluid only, with Basset (1888) providing the time-dependent solution for constant angular velocity. This result has since been generalised to an arbitrary time-dependent angular velocity, $\boldsymbol{\Omega}(t)$, where $t$ is time, for which the torque experienced by the sphere of radius, $a$, is (Kim \& Karrila 2005)

$$
\boldsymbol{T}(t)=-8 \pi \mu a^{3}\left\{\boldsymbol{\Omega}(t)+\frac{1}{3} \int_{-\infty}^{t} \frac{\mathrm{d} \boldsymbol{\Omega}(\tau)}{\mathrm{d} \tau}\left(\frac{1}{\sqrt{\pi \alpha(t-\tau)}}-\mathrm{e}^{\alpha(t-\tau)} \operatorname{erfc}(\sqrt{\alpha(t-\tau)})\right) \mathrm{d} \tau\right\},
$$




\section{J.E. Sader}

where $\mu$ is the fluid's shear viscosity, $\alpha=\mu /\left(\rho a^{2}\right)$ and $\rho$ its density. The term, $\dot{\boldsymbol{\Omega}}(\tau) / \sqrt{\pi \alpha(t-\tau)}$, is identical to the Boussinesq-Basset memory term for the force experienced by a sphere executing rectilinear motion. Superficially, this appears to produce the same long-time transient decay for impulsive start up, varying as $1 / \sqrt{t}$. However, for $\boldsymbol{\Omega}(t)=\boldsymbol{\Omega}_{0} H(t)$, where $H(t)$ is the Heaviside function and $\boldsymbol{\Omega}_{0}$ a constant vector, (1.1) gives the following long-time solution:

$$
T(t)=-8 \pi \mu a^{3} \Omega_{0}\left(1+\frac{1}{6 \sqrt{\pi}(\alpha t)^{3 / 2}}+\cdots\right), \quad \alpha t \gg 1 .
$$

That is, the time-dependent torque approaches steady state at a faster rate, with a time power law of $-3 / 2$ (not $-1 / 2)$. This result, and that for the force experienced by a sphere in rectilinear motion, form part of the vernacular of modern fluid mechanics.

\section{Overview}

Now, 170 years after Stokes first posed his canonical flow problem (quoted above), Taguchi, Tsuji \& Kotera (2021) report its solution for arbitrary degrees of gas rarefaction. This is achieved by solving the linearised Boltzmann-BGK (Bhatnagar, Gross, Krook) equation, which in contrast to the unsteady Stokes equation (a one-dimensional diffusion equation for this problem), represents a formidable challenge in mathematical analysis (Cercignani 1988; Sone 2007).

Why is this important? Virtually all real gas flows contain regions where the continuum hypothesis breaks down. The most obvious region is near a solid surface, where the probability of gas molecules colliding with each other is small relative to the probability of interacting with the surface, i.e. the flow is nearly 'collisionless'. Molecular interactions with the surface can push the gas strongly out of thermal equilibrium, violating a fundamental tenet of continuum theory. A second and less obvious situation arises in start-up problems, such as that studied by Taguchi et al. (2021). Initially, the gas molecules do not have time to collide with each other, producing a collisionless flow everywhere.

A continuum treatment, which the unsteady Stokes equation delivers, cannot capture these non-equilibrium flows. A more fundamental theory is needed, which is provided for by the Boltzmann equation. Rather than requiring specification of a constitutive equation for the gas - standard in continuum treatments - its formulation is based on a statistical mechanical treatment of the molecular gas collisions. The linearised Boltzmann equation (formulated for low Mach number) naturally gives rise to the (linear) unsteady Stokes equation in the continuum limit (Nassios \& Sader 2012; Takata \& Hattori 2012), and captures gas flows under arbitrary degrees of rarefaction at low Reynolds number.

The importance of using a Boltzmann treatment is highlighted by Taguchi et al. (2021) for the heat flow vector; temperature of the sphere is chosen to be identical to that of the stationary gas. Because a purely azimuthal flow arises at low Mach number, a continuum treatment is strictly incompressible and isothermal, and hence predicts zero heat flow. Solution to the linearised Boltzmann equation shows that this prediction is incorrect: non-zero heat flow is always generated near the sphere's surface, despite the temperature not varying throughout the gas. This is beyond the continuum treatment afforded by Fourier's law for heat conduction, which requires a temperature gradient. Following start-up, the heat flow vector points in the same direction as the sphere's motion but then reverses direction as it approaches steady state - with the same $t^{-3 / 2}$ power-law time dependence as for the torque in continuum flow; see $\S 1$.

In fact, Taguchi et al. (2021) show that the long-time decay of all macroscopic flow transients obeys the $t^{-3 / 2}$ scaling law of (1.2), regardless of the degree of gas rarefaction. 
(a)

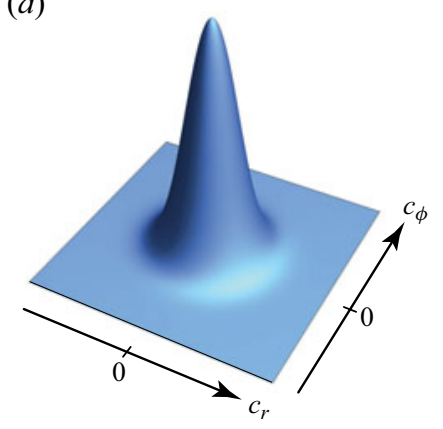

(b)

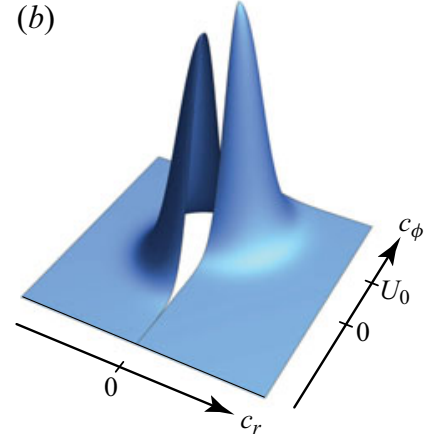

Figure 1. The VDF dependence on radial, $c_{r}$, and azimuthal, $c_{\phi}$, molecular velocities, at the sphere's surface, (a) before, and (b) just after start up. The latter consists of two equilibrium sections, from the surrounding gas and surface, where $U_{0}$ is the sphere's surface speed.

This includes the bulk velocity, stress and heat flow. This feature is not obvious at first sight and shows that a non-continuum flow cannot be identified by observing this scaling law alone. However, the strength of this transient varies with the degree of rarefaction. Perhaps the most important transient from a measurement perspective is that for the torque exerted by the gas on the rotating sphere. Numerical results are given in figure 13 and table 3 of Taguchi et al. (2021) that can be used in practice.

Another characteristic feature of a real low Mach number gas flow, which does not appear in the continuum treatment, is the presence of discontinuities. Rather than directly giving the usual macroscopic variables such as bulk velocity and pressure, the Boltzmann equation gives the distribution of molecular velocities in the gas; as embodied in the 'velocity distribution function' (VDF). Once the VDF of a gas flow is found, all macroscopic variables are generated by its moments. It is within the VDF that discontinuities arise, which can provide insight not possible from the macroscopic variables alone.

Consider motion of the gas just after start up when the flow is strictly collisionless. Any gas molecule impinging on the sphere adsorbs and thermally equilibrates with its surface before being re-emitted; the 'diffuse boundary condition'. This process introduces a discontinuity in the VDF at the surface, about the molecular velocity delineating incoming and outgoing molecules - because incoming molecules are in equilibrium with the surrounding gas, not the surface; see figure 1. The bulk gas velocity is the first moment of the VDF, which in this small time (collisionless) flow gives a value half that of the sphere's surface, regardless of the degree of gas rarefaction. This is the value reported by Taguchi et al. (2021) via solution of the Boltzmann equation. As time evolves, molecular collisions take hold and the degree of slip at the surface reduces. If the gas mean free path is small, this process ultimately leads to the no-slip condition, following which (1.1) applies. Critically, the no-slip condition arises naturally from the diffuse boundary condition and molecular collisions - it is not specified from the outset.

Taguchi et al. (2021) study all VDF discontinuities produced by the rotating sphere. For a steady flow, these occur at the surface (as above) and in the gas along molecular velocity directions making grazing incidence with the surface. The latter discontinuities arise because molecules emitted from the surface and gas have different origins (Yap \& Sader 2016). For the present start-up flow, Taguchi et al. (2021) show that additional VDF discontinuities appear throughout the gas due to the finite time required for molecules to traverse the distance from the surface to a spatial position in the gas. Only molecules 


\section{J.E. Sader}

with very high speed can traverse this finite distance just after start up. This produces transient VDF discontinuities that emerge at infinite molecular speed (ignoring relativistic effects) and move towards zero molecular speed as time evolves, with collisions reducing their strengths. These VDF discontinuities eventually disappear, with those for steady flow remaining. Taguchi et al. (2021) show these additional VDF discontinuities generate abrupt changes in the macroscopic variables near the sphere's surface just after start up, at a time proportional to distance from the surface.

\section{Future}

The theory by Taguchi et al. (2021) predicts phenomena for a problem of Stokes (1851) that are yet to be observed in measurement; for which recent experimental advances may prove useful (Box, Thompson \& Mullin 2015). This theory invokes the BGK collision operator for simplicity. While it erroneously gives a Prandtl number of unity, it can capture features of more realistic collision models (Ladiges \& Sader 2015). The diffuse boundary condition is another simplification. Extension of the theory to more rigorous collision models and realistic gas-surface interactions is an area for future work. Solution to the full nonlinear Boltzmann equation would also allow for intriguing phenomena such as boundary layer collision (Howarth 1951) to be studied. The influence of real gas effects on these and other unsteady nonlinear phenomena remain to be explored.

Declaration of interest. The author reports no conflict of interest.

Author ORCIDs.

(1) John Elie Sader http://orcid.org/0000-0002-7096-0627.

\section{REFERENCES}

BAsset, A.B. 1888 III. On the motion of a sphere in a viscous liquid. Phil. Trans. R. Soc. Lond. A 179, $43-63$.

Box, F., Thompson, A.B. \& Mullin, T. 2015 Torsional oscillations of a sphere in a Stokes flow. Exp. Fluids 56, 209.

Cercignani, C. 1988 The Boltzmann Equation and its Applications. Springer.

HowARTH, L. 1951 Note on the boundary layer on a rotating sphere. Phil. Mag. 42, 1308-1315.

Kim, S. \& Karrila, S.J. 2005 Microhydrodynamics: Principles and Selected Applications. Dover Publications.

LAdigES, D.R. \& SADER, J.E. 2015 Frequency-domain deviational Monte Carlo method for linear oscillatory gas flows. Phys. Fluids 27, 102002.

NASSIOS, J. \& SADER, J.E. 2012 Asymptotic analysis of the Boltzmann-BGK equation for oscillatory flows. J. Fluid Mech. 708, 197-249.

Sone, Y. 2007 Molecular Gas Dynamics: Theory, Techniques, and Applications. Springer.

STOKES, G.G. 1851 On the effect of the internal friction of fluids on the motion of pendulums. Trans. Camb. Phil. Soc. 9, 8-106.

TAguchi, S., Tsuji, T. \& Kotera, M. 2021 Transient behavior of a rarefied gas around a sphere caused by impulsive rotation. J. Fluid Mech.

TAkAtA, S. \& HAtToRi, M. 2012 Asymptotic theory for the time-dependent behavior of a slightly rarefied gas over a smooth solid boundary. J. Stat. Phys. 147, 1182-1215.

YAP, Y.W. \& SADER, J.E. 2016 Sphere oscillating in a rarefied gas. J. Fluid Mech. 794, 109-153. 